\title{
REVIEW
}

\section{Collagenase Treatment in Dupuytren Contractures: A Review of the Current State Versus Future Needs}

Ilse Degreef

Received: June 22, 2015 / Published online: February 3, 2016

(C) The Author(s) 2016. This article is published with open access at Springerlink.com

\section{ABSTRACT}

Dupuytren disease is highly prevalent and the finger contractures can be very extensile, compromising the patients' hand function. To restore full function, contractures have been addressed by cutting the causative strands for nearly 200 years, ever since Baron Guillaume Dupuytren demonstrated his technique at the beginning of the nineteenth century. Surgery can be minimal (fasciotomy) or quite invasive (fasciectomy and even skin replacement). However, in the last decade translational research has introduced the non-surgical technique of enzymatic fasciotomy with collagenase injections. Now, finger contractures can be released with single injections on monthly intervals, to address one joint contracture at a time. However, in hands affected with Dupuytren contractures to the extent that the patient calls for treatment,

Electronic supplementary material The online version of this article (doi:10.1007/s40744-016-0027-1) contains supplementary material, which is available to authorized users.

\section{Degreef $(\bowtie)$}

Orthopedic Department, Hand Surgery, University

Hospitals Leuven, Louvain, Belgium

e-mail: Ilse.degreef@uzleuven.be most often more than one joint is involved. In surgical treatment options all contracted joints are addressed in a single procedure. Nevertheless, extensile surgery withholds inherent risks of complications and intense rehabilitation. Today, the minimally-invasive method with enzymatic fasciotomy by collagenase injection has demonstrated reliable outcomes with few morbidities and early recovery. However, single-site injection is todays' standard procedure and multiple joints are addressed in several sessions with monthly intervals. This triggers a longer recovery and treatment burden in severely affected hands even though surgery is avoided. Therefore, further treatment modalities of collagenase use are explored. Adjustments in the treatment regimes' flexibility and collagenase injections addressing more than one joint contracture simultaneously will improve the burden of multiple sessions and, therefore, enzymatic fasciotomy may become the preferred method in more extensile Dupuytren contractures. In this independent review, the challenge of Dupuytren disease affecting a single versus multiple joints is presented. The pros and cons of collagenase use are weighed, founded by the 
available scientific background. The demands and options for collagenase in future treatment regimens for extensile Dupuytren contractures are discussed.

Keywords: Collagenase fasciotomy; Dupuytren; Fasciectomy; Fasciotomy; Joint contracture; Surgery

\section{INTRODUCTION}

Dupuytren contracture is a highly prevalent hand affection in which contracted fingers compromise hand function. In a recent meta-analysis prevalence up to $30 \%$ in the Western countries was reported, increasing with age [24]. Asymptomatic palmar fibrosis is more common than overt contractures, which require treatment [15]. The standard treatment has been fasciotomy or fasciectomy ever since Baron Guillaume Dupuytren introduced cutting the strands in his the public sessions at the Hotel Dieu in Paris in 1831 [1]. Since then, the pathology has been extensively studied, with historical milestones as the histology stages of Luck in 1959 [27] the identification of the myofibroblast by Gabbiani in 1972 [18]. It is the collagen production of these myofibroblast that holds the finger contractures. This collagen is found more in the cords than in the nodules, which contain mostly myofibroblasts [46]. Both are the targets of surgical treatment: either the cords are cut (fasciotomy) or all of the pathological tissue including nodules is removed (fasciectomy).

Since the cords are collagen rich, understandably the collagenase enzyme has been the topic of research for eventual clinical purposes. Even as long ago as 1971, Hueston [22] instigated the first clinical attempts with enzyme injections. The last decade has become the era of the clinical introduction of clostridium collagenase use in the Dupuytren contracture, after the first successful in vitro report in 1996 [43] and the first clinical open-label study of 35 patients in 2000 [4]. Today's collagenase use is the translational result of many years of basic research and is an example of how surgically treated affections can become treatable or rather controllable without operation.

In this independent review, the focus lies on the urge to extend the collagenase treatment regimen to meet current shortcomings in extensile Dupuytren disease. A literature review is performed on the prevalence of multiple joint affection (the need), the advantages of flexible treatment regimens with simultaneous joint injections (the benefits), the safety issues (the risks), and the comparison with surgical treatment options in multi-strand Dupuytren disease (the assessment). The results are summarized as the current state of the art in collagenase usage in Dupuytren, its limits in severely affected hands, and the requirements or future adjustments needed in the treatment regime to address even severe Dupuytren disease with the non-operative collagenase treatment option.

\section{METHODS}

The literature review was started with a literature search of the electronic database PubMed and Medline. Search terms were Dupuytren disease, collagenase, multiple joints, prevalence, complications, surgery, safety, finger contracture and its plurals or synonyms. Inclusion criteria were set to benefit the goal of this literature review. Articles that present the results of scientific levels 3 and higher (Centre of Evidence-Based Medicine, CEBM) that address the questions of 
this independent review (goals) were included. These goals were mentioned above at the last paragraph of the introduction and focus on the need, the benefits, possible risks and assessment of flexible regimens and multiple joint collagenase treatment options in severely affected hands with Dupuytren disease. This article is based on previously conducted studies and does not involve any new studies of human or animal subjects performed by the author.

\section{RESULTS}

On the prevalence of multiple joint affection in Dupuytren disease, out of 296 articles as a result of the literature search, 15 articles were selected to illustrate the need and benefits for optimization of the single joint and interval multiple session injections of collagenase. On the collagenase treatment in Dupuytren disease, 27 articles out of 100 search results were included to review the options and safety issues of adjusted collagenase treatment regimens. Finally, these options and results were weighed with surgical treatment after a selected number of 21 articles in the search results of 419 .

\section{DISCUSSION}

\section{Collagenase in Dupuytren: Current State of the Art and Encountered Challenges}

Yet incurable, treatment of Dupuytren disease is primarily focused on contracture release to regain finger motion and restore hand function. In the last decade, non-surgical collagenase treatment removed the monopoly of cutting or removing the strands that cause the contractures. The strength of this innovative treatment method is that surgery is avoided, recovery is fast, and the outcome is reliable, with a lower degree of recurrence than needle fasciotomy [33]. The clinical outcome is comparable with surgery (fasciectomy), but collagenase treatment provides a more rapid recovery and is associated with fewer serious adverse events [37, 38, 49].

However, a weakness of the collagenase injection technique is the limit of single joint treatment in one setting with monthly interval repeats if needed to treat more joint contractures. This single-shot injection technique was introduced mostly due to security measures. Toxic dosage limits were set low as a precaution and injection sites were limited to prevent severe immune response such as swelling, hematoma, and lymfangitis.

\section{Treatment Regimen and Its Limits}

Collagenase treatment is usually performed on an outpatient basis. The patient receives his collagen injection on the first day. The second day he returns for a finger manipulation in an effort to break the strands by forcefully extending the finger (usually under local anesthesia) and fit the extension splint. The hand will typically show edema to some extent for 5-10 days; a certain amount of pain and hindrance is expected for about 2 weeks [3]. After care is focused on the recovery of full range of finger motion, which rarely indicates physiotherapy. A night-time splint to maintain full extension for at least 3 months is advised. Collagenase injections need to be planned strategically. The injection is performed on the first day and as prescribed, $24 \mathrm{~h}$ later the manipulation. What's more, the $0.58 \mathrm{mg}$ collagen dose is injected in a single site to address one ray in one session, usually restricted to one joint contracture (either metacarpophalangeal or proximal 
interphalangeal). If multiple ray treatment is required, monthly returning infiltrations and manipulations are needed until sufficient finger motion is achieved and hand function has returned to an acceptable level for the patient. A final restriction is the safe zone: the distal limit of injection sites is the basis of the first phalanx. For safety reasons with respect to the flexor tendon, more distal injections are not advised. Therefore, distal interphalangeal joint contractures may not be addressed.

In total, current treatment regimen with collagenase may restrain surgeons and patients from collagenase treatment in severe Dupuytren disease with multiple extensive ray contractures. Here, the treatment time with recurrent recovery intervals extends the overall recovery time as compared to surgery where all contractures are usually addressed in 1 session. Multiple treatment sessions are obviously a potential burden to the patient and may increase the total treatment cost. The restrictions of the initially prescribed collagenase algorithm are thus increasingly challenged in order to extend its indications and efficiency in severe Dupuytren disease.

\section{Collagenase in Severe Dupuytren Contractures: Pushing Boundaries}

Experience with the collagenase treatment is rapidly increasing. This is reflected in more efficient treatment regimens. First, the organizational issue of having $24 \mathrm{~h}$ in between injection and manipulation has been modified. Mickelson et al. [32] demonstrated in 2014 an optional time span of 7 days in between both sessions without compromising efficiency [23]. The finger extension procedure can be performed the next day or on any occasion within 1 week after the injection, whichever is more convenient [32]. Second, the surgeons experience has increased efficiency of the procedures. Peimer et al. [35] demonstrated that the number of injections per treated joint significantly decreased. In the registration trials, 1.7 injections per joint were required and after 1 year of use, this was reduced to a maximal efficiency of 1.08 injections [35]. More recent data confirmed that most treated joints now require only one injection [48].

However, Coleman et al. [10, 11] demonstrated that many patients present with more than one joint affected and therefore require multiple injections. They calculated a mean (standard deviation) number of affected joints of $3 \pm 2.2$ in their intended to treat population [11]. Multiple ray involvement is seen in more than half of the hands [21, 25]. This means that in the current algorithm of single joint treatment with monthly intervals between injections, the majority of patients will need to undergo multiple sessions in several months. This withholds a lengthy treatment and recovery burden to the patient. The efficiency of collagenase in fixed proximal interphalangeal joint contractures is equal if a second injection session is needed after initial metacarpophalangeal treatment, but the overall treatment is longer [21]. This motivated clinical researchers to explore the option of concurrent multiple strand collagenase treatment in Dupuytren disease to fulfill the important unmet need in the commonly seen multiple digit Dupuytren contractures.

Recent outcome studies report promising results. Gaston et al. [20] demonstrated successful concurrent double-dose injections in one or two cords in a single session. Efficacy was comparable with earlier reports and no adverse events were reported, with the exception of skin laceration in 22\% [29]. Atroshi et al. [2] reported promising outcomes in higher dosage injections with a technique modification allowing 
multiple joint corrections in a single session. Again, more skin tears were encountered (in $40 \%)$. It is unclear whether the double dose is the cause of these skin lacerations or rather the more significant contracture release is. Hence, skin tears are also seen in needle fasciotomy, without enzyme injection in $27 \%$ of the patients [39]. Another option is to use the whole bottle of collagenase clostridium histolyticum $(0.78 \mathrm{mg}$ instead of $0.58 \mathrm{mg})$ : this was reported by Verheyden [45] as another efficient way to increase the dosage and safely treat multi-cord affections in a single session in 144 patients.

However, if multiple strand treatment is considered, risks and benefits need to be outweighed and scientific support for this clinical research needs to be explored.

\section{Risks}

The risks of injecting collagenase are predominantly injection-related and transient: skin rupture, tendon rupture, swelling and edema, hematoma, pain, lymfangitis, fever, and pruritus [36]. Severe allergic reactions and anaphylactic shock are possible but not one case has been reported up until now. On the other hand, antibodies to collagenase may develop after several treatments, raising concerns about safety and efficacy as a result of sensitization from repeated exposures. The development of recombinant enzyme treatment methods was initially introduced for treating lysosomal storage disorders. Now, the collagen-based disorder of Dupuytren disease is treated with the collagenase clostridium hystolyticum. Although enzyme treatment may hence carry the risk of immunological response certainly in repeated long-term treatment logarithms, safety findings and adverse event reports and surely problems of immunogenicity remain mild in general [5]. Gajendran et al. [19] reported good results in a patient receiving 12 dosages in 15 injections over a 4-year period in 2014. They compare the evolution and increasing knowledge with the botulinum toxin injection experience and future adjustments of the formula to prevent blocking antibodies in some patients may be required 1 day.

\section{Benefits}

If ascertained safe and efficient, treating two joints simultaneously with collagenase injections in a single session will obviously benefit the patient and treating surgeon in different ways. First, overall treatment time will be reduced significantly. Treating two joints in one session, means restoring motion in at least one complete finger in a maximum of one treatment week, where in two monthly interval sessions, it would mean a total treatment time of four to 6 weeks. What is more, optimized injection techniques add to this benefit. For instance, a y-type strand causing more than one finger contracture can be solved with strategic injections with even more extensile corrections, in which more fingers (including two to even six joints) are addressed in a single treatment session [30]. This obviously precludes a lower working incapacity for the patient with a lower healthcare cost, implies less time to treat, and higher patient satisfaction with immediate results $[10,11]$. On top of fewer treatment sessions and faster rehabilitation, one must also consider the benefit in more efficient orthotic devices without secondary adjustments, since every session changes the configuration of the hand [31]. Furthermore, if therapy is indicated after collagenase treatment, as required in more severe contractures mostly of interphalangeal joints, the rehabilitation period will equally be reduced as overall injection sessions [42]. 
Last, the anatomical restriction of injection sites that excludes the distal interphalangeal joint contractures was sporadically challenged in off-label use in limited cases by different surgeons, including the author. These more rare contractures can be successfully addressed with a $0.1 \mathrm{mg}$ injection in the lateral cord at the proximal interphalangeal (PIP) joint.

Obviously, the increasing efficiency in collagenase injections with fewer treatment sessions and increased gain in finger motion will benefit more patients suffering from severe Dupuytren disease. This evolution may therefore reduce the need for elaborate surgery with its inherent risks in the more challenging Dupuytren contractures.

\section{Collagenase in Future Treatment Regime for Dupuytren Disease}

Collagenase now has become common practice in numerous hand surgery practices for Dupuytren treatment and its position in the regimen is yet evolutionary [20,47].

The optimization of collagenase injections in Dupuytren contractures may reduce surgery even more in the overall treatment regime. Collagenase treatment can be extended to more severe Dupuytren disease. In severely affected hands, extensile surgery carries a higher risk for complications in up to $46 \%$ of the patients $[8,12,14,26,28]$. Digital nerve or artery injuries are even 10 times more common in recurrent disease than in primary surgery [12, 17, 41].

Local health economic differences may influence treatment regimes, although generally the total social cost of collagenase treatment of Dupuytren contracture is lower than surgery $[6,9,13,31,38,40]$. In countries such as Belgium, where healthcare reimbursement is limited to a number of injections per hand after simple cost-benefit analysis, the optimization of collagenase injection techniques is essential to offer more patients the option of enzymatic fasciotomy to avoid or postpone surgery [38].

Recurrence risk after collagenase treatment is not lower than in surgery, with similar reports varying between $4 \%$ after 1 year to $47 \%$ in 5 years. These results are comparable with recurrence reports in surgical outcome studies with a variety of recurrence reports ranging from 0 to $71 \%$, depending on definition and time of follow-up [7, 29, 34]. Surgical technique does not influence recurrence rates, although needle fasciotomy has a particularly high recurrence [7, 44]. In recurrence after collagenase treatment, repeat injections or even surgery remain viable options to treat recurrent contractures $[16,19]$.

\section{CONCLUSIONS}

Since the collagenase technique was introduced in the treatment regime for Dupuytren disease, experience has built, injection efficiency has increased, and satisfaction of both patient and surgeon is markedly high. For safety reasons, single joint injections (single site) were initially advised. Recently, exploratory studies on multiple injection sites demonstrated promising outcomes. Today, multiple joint corrections in a single session and even complete multiple digit treatment have become an option. Collagenase injection technique optimization is needed to limit the time to treat, working incapacity, healthcare costs, and burden of multiple repeated treatment sessions. Ultimately, collagenase treatment will preferably result in maximal restoration of the hand function in minimal numbers of treatment sessions, even in severe Dupuytren disease. This may minimize the 
burden of surgery to treat the finger contractures in Dupuytren disease, taken that not any treatment will cure the patient indefinitely and recurrent contractures are highly prevalent.

\section{ACKNOWLEDGMENTS}

No funding or sponsorship was received publication of this article. The named author meets the International Committee of Medical Journal Editors (ICMJE) criteria for authorship for this manuscript, takes responsibility for the integrity of the work as a whole, and has given final approval for the version to be published.

Disclosures. Ilse Degreef has nothing to disclose.

Compliance with ethics guidelines. This article is based on previously conducted studies and does not involve any new studies of human or animal subjects performed by the author.

Open Access. This article is distributed under the terms of the Creative Commons AttributionNonCommercial 4.0 International License (http://creativecommons.org/licenses/by-nc/4. $0 /$ ), which permits any noncommercial use, distribution, and reproduction in any medium, provided you give appropriate credit to the original author(s) and the source, provide a link to the Creative Commons license, and indicate if changes were made.

\section{REFERENCES}

1. Alexandre D. Report on the surgical clinic at the Hotel-Dieu. 1831. J Hand Surg Br. 2005;30(6):546-50.
2. Atroshi I, Nordenskjöld J, Lauritzson A, Ahlgren E, Waldau J, Waldén M. Collagenase treatment of Dupuytren's contracture using a modified injection method: a prospective cohort study of skin tears in 164 hands, including short-term outcome. Acta Orthop. 2015;86(3):310-5. doi:10.3109/17453674. 2015.1019782.

3. Badalamente MA, Hurst LC, Benhaim P, Cohen BM. Efficacy and safety of collagenase clostridium histolyticum in the treatment of proximal interphalangeal joints in dupuytren contracture: combined analysis of 4 phase 3 clinical trials. J Hand Surg Am. 2015;40(5):975-83. doi:10.1016/ j.jhsa.2015.02.018. (Epub 2015 Apr 2. PubMed PMID: 25843533).

4. Badalamente MA, Hurst LC. Enzyme injection as nonsurgical treatment of Dupuytren's disease. J Hand Surg Am. 2000;25(4):629-36.

5. Baldo BA. Enzymes approved for human therapy: indications, mechanisms and adverse effects. BioDrugs. 2015;29(1):31-55. doi:10.1007/s40259015-0116-7.

6. Baltzer H, Binhammer PA. Cost-effectiveness in the management of Dupuytren's contracture. A Canadian cost-utility analysis of current and future management strategies. Bone Joint J. 2013;95-B(8):1094-100. doi:10.1302/0301-620X. $95 \mathrm{~B} 8.31822$.

7. Becker GW, Davis TRC. The outcome of surgical treatments for primary Dupuytren's disease-a systematic review. J Hand Surg Br. 2010;35(8):623-6.

8. Bulstrode NW, Jemec B, Smith PJ. The complications of Dupuytren's contracture surgery. J Hand Surg [Am]. 2005;30:1021-5.

9. Chen NC, Shauver MJ, Chung KC. Cost-effectiveness of open partial fasciectomy, needle aponeurotomy, and collagenase injection for dupuytren contracture. J Hand Surg Am. 2011;36(11):1826-1834.e32. doi:10.1016/j.jhsa. 2011.08.004. (Epub 2011 Oct 5).

10. Coleman S, Gilpin D, Kaplan FT, Houston A, Kaufman GJ, Cohen BM, Jones N, Tursi JP. Efficacy and safety of concurrent collagenase clostridium histolyticum injections for multiple Dupuytren contractures. J Hand Surg Am. 2014;39(1):57-64. doi:10.1016/j.jhsa.2013.10.002 (Epub 2013 Dec 4).

11. Coleman S., Gilpin D., Tursi J., Kaufman G., Jones N., Cohen B. Multiple concurrent collagenase clostridium histolyticum injections to Dupuytren's cords: an exploratory study BMC Musculoskelet Disord, 13 (2012), p. 61 (12). 
12. Denkler K. Surgical complications associated with fasciectomy for Dupuytren's disease: a 20-year review of the English literature. Eplasty. 2010;10:e15.

13. Dias J, Bainbridge C, Leclercq C, Gerber RA, Guerin D, Cappelleri JC, Szczypa PP, Dahlin LB. Surgical management of Dupuytren's contracture in Europe: regional analysis of a surgeon survey and patient chart review. Int J Clin Pract. 2013;67(3):271-81. doi:10.1111/ijcp.12106.

14. Dias JJ, Braybrooke J. Dupuytren's contracture: an audit of the outcomes of surgery. J Hand Surg [Br]. 2006;31:514-21.

15. Diep GK, Agel J, Adams JE. Prevalence of palmar fibromatosis with and without contracture in asymptomatic patients. J Plast Surg Hand Surg. 2015;49(4):247-50.

16. Eberlin KR, Kobraei EM, Nyame TT, Bloom JM, Upton J 3rd. Salvage palmar fasciectomy after initial treatment with collagenase clostridium histolyticum. Plast Reconstr Surg. 2015; 135(6):1000e-6e. doi:10.1097/PRS.000000000000 1282.

17. Ebskov LB, Boeckstyns MEH, Sorensen AI, Haugegaard M. Day care surgery for advanced Dupuytren's contracture. J Hand Surg [Br]. 1997;22:191-2.

18. Gabbiani G, Majno G. Dupuytren's contracture: fibroblast contraction? An ultrastructural study. Am J Pathol. 1972;66(1):131-46. PubMed PMID: 5009249; PubMed Central PMCID: PMC2032479.

19. Gajendran VK, Hentz V, Kenney D, Curtin CM. Multiple collagenase injections are safe for treatment of Dupuytren's contractures. Orthopedics. 2014;37(7):e657-60. doi:10.3928/ 01477447-20140626-64.

20. Gaston RG, Larsen SE, Pess GM, Coleman S, Dean B, Cohen BM, Kaufman GJ, Tursi JP, Hurst LC. The efficacy and safety of concurrent collagenase clostridium histolyticum injections for 2 Dupuytren contractures in the same hand: a prospective. Multicenter study. J Hand Surg Am. 2015;40(10):1963-71. doi:10.1016/j.jhsa.2015.06. 099.E.

21. Hayton MJ, Bayat A, Chapman DS, Gerber RA, Szczypa PP. Isolated and spontaneous correction of proximal interphalangeal joint contractures in Dupuytren's disease: an exploratory analysis of the efficacy and safety of collagenase Clostridium histolyticum. Clin Drug Investig. 2013;33(12):905-12.

22. Hueston JT. Enzymic fasciotomy. Hand. 1971;3(1): 38-40.
23. Kaplan FT, Badalamente MA, Hurst LC, Merrell GA, Pahk R. Delayed manipulation after collagenase clostridium histolyticum injection for Dupuytren contracture. Hand (N Y). 2015;10(3):578-82. doi:10.1007/s11552-014-9714-y.

24. Lanting R, Broekstra DC, Werker PM, van den Heuvel ER. A systematic review and meta-analysis on the prevalence of Dupuytren disease in the general population of Western countries. Plast Reconstr Surg. 2014;133(3):593-603.

25. Lanting R, Nooraee N, Werker PM, van den Heuvel ER. Patterns of Dupuytren disease in fingers: studying correlations with a multivariate ordinal logit model. Plast Reconstr Surg. 2014; 134(3):483-90. doi:10.1007/s40261-013-0139-0.

26. Loos B, Puschkin V, Horch RE. 50 years experience with Dupuytren's contracture in the Erlangen University Hospital-a retrospective analysis of 2919 operated hands from 1956 to 2006. BMC Musculoskelet Disord. 2007;8:60.

27. Luck JV. Dupuytren's contracture; a new concept of the pathogenesis correlated with surgical management. J Bone Joint Surg Am. 1959;41-A(4):635-64. PubMed PMID: 13664703.

28. McFarlane RM, McGrouther DA. Complications and their management. In: McFarlane RM, McGrouther DA, Flint MH, editors. Dupuytren's disease. New York: Churchill Livingstone; 2009. p. 377-382.

29. McGrouther DA, Jenkins A, Brown S, Gerber RA, Szczypa P, Cohen B. The efficacy and safety of collagenase clostridium histolyticum in the treatment of patients with moderate Dupuytren's contracture. Curr Med Res Opin. 2014;30(4):733-9. doi:10.1185/03007995.2013.874990.

30. Meals RA, Hentz VR. Technical tips for collagenase injection treatment for Dupuytren contracture. J Hand Surg Am. 2014;39(6):1195-200.e2. doi:10. 1016/j.jhsa.2014.03.016. PubMed PMID: 24862115.

31. Mehta S, Belcher HJ. A single-centre cost comparison analysis of collagenase injection versus surgical fasciectomy for Dupuytren's contracture of the hand. J Plast Reconstr Aesthet Surg. 2014;67(3):368-72. doi:10.1016/j.bjps.2013. 12.030 (Epub 2014 Jan 4).

32. Mickelson DT, Noland SS, Watt AJ, Kollitz KM, Vedder NB, Huang JI. Prospective randomized controlled trial comparing 1-versus 7-day manipulation following collagenase injection for dupuytren contracture. J Hand Surg Am. 2014;39(10):1933-41.e1. doi:10.1016/j.jhsa.2014. 07.010. PubMed PMID: 25194768. (Epub 2014 Sep 4). 
33. Nydick JA, Olliff BW, Garcia MJ, Hess AV, Stone JD. A comparison of percutaneous needle fasciotomy and collagenase injection for dupuytren disease. J Hand Surg Am. 2013;38(12):2377-80. doi:10. 1016/j.jhsa.2013.08.096.

34. Peimer CA, Blazar P, Coleman S, Kaplan FT, Smith $\mathrm{T}$, Lindau T. Dupuytren contracture recurrence following treatment with collagenase Clostridium histolyticum (CORDLESS [collagenase option for reduction of dupuytren long-term evaluation of safety study]): 5-year data. J Hand Surg Am. 2015;40(8):1597-605. doi:10.1016/j.jhsa.2015.04. 036.

35. Peimer CA, Skodny P, Mackowiak JI. Collagenase clostridium histolyticum for dupuytren contracture: patterns of use and effectiveness in clinical practice. J Hand Surg Am. 2013;38(12):2370-6. doi:10.1016/j.jhsa.2013.08. 114 Epub 2013 Oct 17.

36. Peimer CA, Wilbrand S, Gerber RA, Chapman D, Szczypa PP. Safety and tolerability of collagenase Clostridium histolyticum and fasciectomy for Dupuytren's contracture. J Hand Surg Eur. 2015;40(2):141-9. doi:10.1177/1753193414528843.

37. Povlsen B, Povlsen SD. What is the better treatment for single digit dupuytren's contracture: surgical release or collagenase clostridium histolyticum (Xiapex) injection? Hand Surg. 2014;19(3): 389-92.

38. Povlsen B, Shields AM, Bhabra GS. Resource utilisation associated with single digit Dupuytren's contracture treated with either surgery or injection of collagenase Clostridium histolyticum. Hand Surg. 2014;19(2):205-9. doi:10.1142/S021881041450021X.

39. Salhi S, Cardin-Langlois E, Luc M. Percutaneous fasciotomy for the treatment of Dupuytren's disease-a systematic review. Hand (N Y). 2011;6(4):349-55. doi:10.1007/s11552-011-9355-3.

40. Sanjuan Cerveró R, Franco Ferrando N, Poquet Jornet J. Use of resources and costs associated with the treatment of Dupuytren's contracture at an orthopedics and traumatology surgery department in Denia (Spain): collagenase Clostridium hystolyticum versus subtotal fasciectomy. BMC Musculoskelet Disord. 2013;14:293. doi:10.1186/ 1471-2474-14-293.

41. Sennwald GR. Fasciectomy for treatment of Dupuytren's disease and early complications. J Hand Surg [Am]. 1990;15:755-61.
42. Skirven TM, Bachoura A, Jacoby SM, Culp RW, Osterman AL. The effect of a therapy protocol for increasing correction of severely contracted proximal interphalangeal joints caused by dupuytren disease and treated with collagenase injection. J Hand Surg Am. 2013;38(4):684-9. doi:10.1016/j.jhsa.2013.01.038. PubMed PMID: 23474162. (Epub 2013 Mar 6).

43. Starkweather KD, Lattuga S, Hurst LC, Badalamente MA, Guilak F, Sampson SP, Dowd A, Wisch D. Collagenase in the treatment of Dupuytren's disease: an in vitro study. J Hand Surg Am. 1996;21(3):490-5.

44. van Rijssen AL, Ter Linden H, Werker PM. Five-year results of a randomized clinical trial on treatment in Dupuytren's disease: percutaneous needle fasciotomy versus limited fasciectomy. Plast Reconstr Surg. 2012;129(2):469-77.

45. Verheyden JR. Early outcomes of a sequential series of 144 patients with Dupuytren's contracture treated by collagenase injection using an increased dose, multi-cord technique. J Hand Surg Eur. 2015;40(2):133-40. doi:10.1177/1753193414530590.

46. Verjee LS, Midwood K, Davidson D, Essex D, Sandison A, Nanchahal J. Myofibroblast distribution in Dupuytren's cords: correlation with digital contracture. J Hand Surg Am. 2009;34(10):1785-94.

47. Warwick D, Arner M, Pajardi G, Reichert B, Szabo Z, Masmejean EH, Fores J, Chapman DS, Gerber RA, Huard F, Seghouani A, Szczypa PP, POINT X Investigators. Collagenase Clostridium histolyticum in patients with Dupuytren's contracture: results from POINT $X$, an open-label study of clinical and patient-reported outcomes. J Hand Surg Eur. 2015;40(2):124-32. doi:10.1177/1753193413519926.

48. Witthaut J, Jones G, Skrepnik N, Kushner H, Houston A, Lindau TR. Efficacy and safety of collagenase clostridium histolyticum, a nonsurgical treatment for adults with Dupuytren's contracture: short-term results from two open-label studies, in the US (JOINT I) and Australia and Europe (JOINT II). J Hand Surg Am. 2013;38(1):2-11.

49. Zhou C, Hovius SE, Slijper HP, Feitz R, Van Nieuwenhoven CA, Pieters AJ, Selles RW. Collagenase Clostridium histolyticum versus limited fasciectomy for Dupuytren's contracture: outcomes from a multicenter propensity score matched study. Plast Reconstr Surg. 2015;136(1):87-97. doi:10. 1097/PRS.0000000000001320. 